\title{
ChaRACTERIZATION OF DIRECTED DIFFUSION Protocol in Wireless SENSOR NETWORK
}

\author{
Mohammad Abdus Salam and Tanjima Ferdous \\ Department of Computer Science \\ Southern University, Baton Rouge, LA 70813, USA
}

\begin{abstract}
Wireless sensor network (WSN) has enormous applications in many places for monitoring the environments of importance. Sensor nodes are capable of sensing, computing, and communicating. These sensor nodes are energy constraint and operated by batteries. Since energy consumption is an important issue of WSN, there have been many energy-efficient protocols proposed for the WSN. Directed diffusion (DD) is a datacentric protocol that focuses on the energy efficiency of the networks. Since the first proposal of DD protocol by Deborah, there have been various versions of DD protocols proposed by many scientists across the globe. These upgraded versions of DD protocols add on various features to the original DD protocol such as energy, scalability, network lifetime, security, reliability, and mobility. In this paper, we discuss and classify various characteristics of themost populardirected diffusion protocols that have been proposed over couple of years.
\end{abstract}

\section{KEYWORDS}

Wireless sensor networks, security, directed diffusion algorithm, cryptography, synchrony

\section{INTRODUCTION}

Wireless sensor networks consist of low-cost energy-constraint sensor nodes. These sensor nodes have sensing, data processing, and communicating capabilities. WSN promises researchers a powerful instrument for observing various phenomena and it has been deployed in many application domains such as habitat monitoring, and scientific exploration. WSN nodes are prone to be failure due to energy depletion, hardware failure, communication link errors, malicious attack, and so on. Therefore, the reliability and accuracy of individual sensor nodes' reading is very crucial. Many protocols have been developed to ensure secure data routing and aggregation for WSN throughout the past couple of years. Many researchers have also addressed the potential of collaboration among sensor nodes in data gathering and aggregating. However, sensor nodes are constrained in energy supply, computing capability, and bandwidth. Thus, innovative routing techniques have an important consideration for the reduction of these constraints. Routing in WSN is very challenging and has distinguishing characteristics compared to other wireless networks.

In our paper, we mainly focus on the directed diffusionbased routing techniques. The data gathering of these protocols can be characterized as the systematic collection and transmission of sensed data from multiple sensor nodes and processing them at a remote location for final decision-making. On the other hand, data aggregation is a process of aggregating the data from multiple sensor nodes to eliminate redundant transmission and provide fused information to the base station. Security in data gathering and aggregation is an important design issue for wireless sensor networks. Secure data aggregation of information is a challenging task when sensors are deployed in hostile environments and are susceptible to physical attacks.

DOI : 10.5121/ijwmn.2014.6301 
International Journal of Wireless \& Mobile Networks (IJWMN) Vol. 6, No. 3, June 2014

\section{DIRECTED DIFFUSION BASED ROUTING PROTOCOLS}

In this section, we discuss directed diffusion based routing protocols for WSN. In this paper, we describe and classify some directed diffusion based routing protocols which discuss the secure data aggregation problem and also explain some of the main issues involved in implementing security in sensor networks.

\subsection{Agent-based Directed Diffusion Sensor Networks [1]}

Agent-based Directed Diffusion(AbDD) is a mobile agent-based version of directed diffusion that increases the operation efficiency. In this paper, two potential improvements to AbDDare proposed by introducing spatial distribution modeling and optimizing the number of mobile agents employed. The work here is inspired from a property of the individual behaviors of the spatial distribution patterns of insect populations, emergent at the population level. An insect population can be projected as a multi-modal wireless sensor network in which each of the sensors are capable of processing multimodal signals (e.g., auditory, visual, and chemosensory) from environment and their peers. Considering this spatial property, Zhanshanet al optimized the network characteristics by considering the behavior of sensors. This paper focuses on two things:(1) explore spatial models that can characterize the AbDD and (2) explore the optimum number of agents for an AbDD network based on the models.

\subsection{A Distributed Virtual MIMO Scheme Using Cross Layer Design for Wireless Sensor Networks [2]}

In this paper, a protocol called "novel distributed virtual Multiple-Input-Multiple-Output (MIMO) transmission scheme based on the directed diffusion routing protocol" has been proposed which provides a cross-layer design. Here, in network layer, the directed diffusion paradigm is used, while in physical layer a virtual MIMO technique is used. In directed diffusion, we find some characteristics of it such as data-centric, application-aware and in-network processing. Now, to improve the reliability \& efficiency issues of the network the virtual MIMO technique is used on the physical layer. Virtual MIMO techniques are proposed to make the sensors in the wireless sensor network act as a multi-antenna array through interchange of messages [2]. Virtual MIMO technique gives the opportunity to use appropriate gradient information as the antenna selection criterion while directed diffusion provides local information interaction that balances the network load. Another significance of using virtual MIMO along with DD is to make the network capable to adapt the topological change and have stronger stability and scalability.

\subsection{A Gradient Reconfiguration Mechanism of Directed Diffusion in Wireless Sensor Networks [3]}

In [3], an important issue is discussed that is node mobility. When the node is moving, the network gets partitions and some nodes lose their connection to the network. This challenges the regular flow of data streaming throughout the network. Being concerned about this issue, the authors proposed a protocol called Mobility and Energy-Efficiency based Directed Diffusion (MEDD). Whenever a node moves it would be able to localize the gradient (a feature of Directed Diffusion) reconfiguration and could reconstruct its connection with the network. The MEDD has three basic contributions: (a)a gradient model is designed to considers residual energy of node and the numbers of hops from sink to an intermediate node, to decrease the network overhead, (b) a mechanism is developed to make a moving node able to localize the gradient reconfiguration by messages exchange among its neighbors, and (c)a performance analysis of gradient scheme for the increase of total number of nodes in the network. 
International Journal of Wireless \& Mobile Networks (IJWMN) Vol. 6, No. 3, June 2014

\subsection{A hierarchical scheme for data aggregation in sensor network [4]}

This paper [4] is an enhanced version of directed diffusion. It claims an effective enhancement of directed diffusion which will provide much betterment. The scheme consists of four stages. First stage is the hierarchy construction of the nodes. In this stage, a node sends an interest message to all its neighbors. Unlike the directed diffusion, interest message will have an extra attribute named 'level'. This field is going to sort out which node is in which level. A node receiving an interest with a level $\mathrm{N}$, will denote itself as in $\mathrm{N}^{\text {th }}$ level and will record the sender as its parent. Same process will go till the level $\mathrm{N}+2$. Second stage is attribute establishment. In hierarchical tree building, though there were several parents, a node will choose only one parent to transmit the real data. Unlike the directed diffusion, in this stage, this scheme is going to add an extra field in the exploratory data which will identify the data source. Third stage is the data aggregation. In this stage, the sink reinforces the interest to get the real data. The special procedure followed here is called per hop aggregation because data are opportunistically aggregated by each parent. Fourth stage is data distribution. This stage illustrates how data is drawn back in multiple sinks. With multiple sinks, each sink will have its own hierarchical tree with separate interest. This algorithm is a better implementation of directed diffusion by saving 50\% of transmission energy and it provides greater reliability.

\subsection{A Hybrid Protocol for Wireless Sensor Network [5]}

In [5], Haque proposed the hybrid of two different types of protocols, one is directed diffusion and another is rumor routing. The author mentioned them as two opposite kind of protocol in sense that Directed Diffusion initiates the protocol from the sink, which propagates interest messages through the network in search of a matching event, while, in rumor routing, nodes themselves propagate the rumor of an event. There would be some common nodes concerned with both of the protocols, which would be using their gradients (like Directed Diffusion). They also get the agent packet carrying the rumors (like Rumor Routing). They forward those agent packets along the gradientstraight to the sink. This is how, an optimized combination is achieved. The protocol is totally distributed in nature. This also features limited flooding, less probability of missing events, adaptive hop count readjustment and maximum utilization of gradients.

\subsection{A Proposal for Energy Optimization in wireless sensor networks using a geo-cast approach [6]}

In [6], Araujo et al achieved energy optimization by modifying directed diffusion when there is an occurrence of failure. By evaluating two geo-cast routing methods, a new protocol is developed to repair broken paths by constructing a new routing tree. These two geo-cast methods are the rectangular geocast routing method (RGRM) and the cone geo-cast routing method (CGRM). The purpose of using this geo-cast approach is to reduce the number of sent and received control messages. This reduction is achieved through the construction of a newrouting tree, which is limited and directed towards a specific region of the network where the network failure occurs. The proposal modifies directed diffusion in two ways: the first one proposes the use of a method of a geo-cast routing whilst the second one consists of a mechanism for selection of routes. These modifications aim to reduce the flooding caused by the directed diffusion in order to recover broken paths and to determine the best route to deliver the collected event.

\subsection{A Radio-Aware Routing Algorithm for Reliable Directed Diffusion in Lossy Wireless Sensor Networks [7]}

Kim et al have pointed out some weakness of directed diffusion, which usually occurs due to unexpected network errors [7]. Such weaknesses are lossy network environment, suffering from low data delivery rates, and excessive energy consumption from frequent flooding of control packets. In order to address these problems, the authors proposed a radio-aware routing algorithm 
to improve the reliability of directed diffusion in a lossy wireless sensor network. The algorithm is designed to stay aware of the network status by getting radio information from MAC and PHY layers by creating a cross-layer. This cross layer also can provide status information of link quality or transmission errors of communication links. All these information together provides a space to the network to decide and choose the best available route to propagate data reliably.

\subsection{A routing metric for load balancing considering energy and delay constraints in wireless sensor network [8]}

In this paper, Dehghani et al concentrated on the increment of network reliability. In order to enhance network reliability as well as energy balancing, a routing metric is introduced which will contribute to select intermediate nodes from source to sink based on remaining energy in the node, buffer capacity, transmission delay, and link quality. This paper modified a routing metric and applying it in the field of directed diffusion. Here directed diffusion is extended using a filter called packet reception rate.

\subsection{A Secure Directed Diffusion Routing Protocol for Wireless Sensor Networks [9]}

The concentration of this paper [9] is the security issue. In the basis of directed diffusion, another protocol called the $\mu$ TESLA (micro Timed, Efficient, Streaming, Loss tolerant Authentication) is utilized in order to authenticate acknowledgement messages sent from the sink to the source. This is to ensure the delivery of data-event messages. To verify the security issue, this protocol is evaluated and conducted against black hole and acknowledgement-spoofing attacks. Simulation study showed that this secure routing protocol achieves better event-deliveryand event-dropping ratios compared to directed diffusion. The protocol flows according to some basic steps: 1) Secret-key setup and broadcasting phase, 2) Interest propagation phase, 3) Path establishment and Reinforcement phase, 4) Data-event delivery and authenticated acknowledgement phase, and 5) Disclosed key and buffered ACK-packets authentication phase. However, it results higher cost in the mean dissipated energy and average delay in some situations because of acknowledgements and authentication processes for delivered events as well as because of the retransmissions of non-acknowledged events.

\subsection{A Self-Stabilizing Directed Diffusion Protocol for Sensor Networks [10]}

Bein et al proposed a self-stabilizing communication algorithm [10]. This protocol is established on the platform of directed diffusion indicating that the initiated interest from a sink/initiator node is broadcasted in the network, and the positive answers from the sensors are sent back to the initiator following a Shortest-Path-Tree (SPT) construction which is rooted at the initiator. The objective of the development of this protocol is to establish reliable communication in the network in a finite number of steps. It gives the network the freedom to have any number of initiators and any number of different requests at a time per initiator. This protocol guarantees the convergence of the intended behavior within finite time regardless of the system starting. The system is also claimed to cope withtopology changes as well.

\subsection{A Study on a Sequenced Directed Diffusion Algorithm for Sensor Networks} [11]

In this paper a routing protocol has been proposed that improved the energy efficiency of basic Directed Diffusion. It emphasizes on the data interest and exploratory phase of DD which uses the flooding method while setting connection paths. It is found that this flooding process consumes a lot extra energy. This paper proposes a new sensor network routing protocol named sequenced directed diffusion with a threshold control that offers energy efficiency. 


\subsection{Adaptive directed diffusion routing in wireless sensor network based on application [12]}

This paper emphasizes on the fact thatperformance of directed diffusion can result in drastically poor when mismatched to applications. So an application based adaptive directed diffusion routing protocol for wireless sensor networks is proposed in this paper. The protocol proposed over here is called ADDRA (Adaptive directed diffusion routing in wireless sensor network based on application). ADDRA is defined as application aware as it can switch adaptively from normal model to push model according to current environmental scenarios. This paper shows comparative simulation result with other enhanced versions of directed diffusion.

\subsection{Algorithm for optimizing energy use and path resilience in sensor networks [13]}

The basic part of directed diffusion is flooding which is costly as well as energy consuming. This matter also provides chances of node failures which could cause path failure. So the algorithm should be resilient to path failure and should have alternative extra paths. But maintain extra path is energy consuming too. This paper solves this problem by designing a protocol, which is a better alternative to the braided multi-path algorithm presented in [11]. This algorithm reduces the maintenance overhead associated with multiple paths and increases their resilience to node failure. The simulation results showed that this algorithm balances the energy consumption better.

\subsection{An efficient gradient mechanism of Directed Diffusion in Wireless Sensor Network [14]}

In wireless sensor network the requirements are sometimes sophisticated. It may ask for timely deliver for which real-time has also become needed for routing. According to this paper, directed diffusion is not able to neither support time-sensitive traffic nor perform balance of energy consumption to increase network lifetime, solving which is an objective of the algorithm proposed here. To solve this issue, a new kind of gradient mechanism is introduced that considers residual energy of node and the number of hops from the sink to an intermediate node synchronously. It discovers an optimal path in sensor networks for data propagation.

\subsection{An energy-aware broadcast scheme for directed diffusion in wireless sensor network [15]}

One of the main features of directed diffusion is broadcast or flooding procedure used to disseminate the interest. But this could create broadcast storm problems, excessive energyconsumption. This paper works on different broadcasting method on the field of directed diffusion and proposes an energy-aware broadcasting scheme. This scheme combines the advantages of distance-based and counter-based broadcast schemes as well as considers the residual energy of sensor node. Simulation results showed that this scheme provides some advantages such as simplicity, efficiency and scalability, more energy balancing, and longer network lifetime than otherschemes.

\subsection{An energy aware spanning tree algorithm for data aggregation in wireless sensor network [16]}

This paper proposes an energy aware spanning tree algorithm which is called E-Span. This is a tree based structural algorithm which selects the shortest path to take as a route for data propagation within the network. This protocol facilitates the source node to aggregate data within the event region. The source node will be having the highest residual energy and will be chosen as root. If there are other source nodes, those would be choosing their parent nodes among their neighbors based on the residual energy and distance to the root. This algorithm has utilized 
directed diffusion by implementing it as its underlying routing platform. The simulation has proven that this algorithm is capable to extend the lifetime of the network.

\subsection{An Energy Efficient Directed Diffusion Model for Wireless Sensor Networks [17]}

This paper emphasizes on a fact that the flooding procedure followed in interest diffusion phase will inevitably bring about heavy traffic load and affect seriously transmission performance of network. To lessen this flaw an improved ant colony algorithm is presented and applied on the platform of DD, which is called IACA-DD. To mitigate the energy consumption of the whole network, this IACA-DD algorithm focuses on the remainder energy of the nodes and the gradients which is considered as the pheromone of ants. It improves the way of flooding interests. Experiments and simulation studies prove that this protocol is capable to prolong the network lifetime.

\subsection{Load-balance Directed Diffusion in Wireless Sensor Networks [18]}

This paper talks about the power consumption of the nodes considering in terms of load in the wireless sensor network. In DD, interest is disseminated through flooding process and path gradient is calculated as well as recalculated (while sending exploratory data) using the interest message. Both of flooding and gradient calculating are power consuming. Besides if the transmission period of interest message takes long than load imbalance occurs and network lifetime is also reduced. Considering these, this paper proposes a load-balanced directed diffusion paradigm that presents a new path switching mechanism to automatically switch to different route based on the current gradient information to achieve load balance.

\subsection{Mobile Agent-Based Directed Diffusion in Wireless Sensor Networks [19]}

This paper points on a fact that in network environments where the source nodes are close to each other and generate a lot of sensory data traffic with redundancy, transmitting all sensed data by individual nodes does waste the limited wireless bandwidth as well as consumes a lot of battery energy. To lessen this problem, this paper proposes a routing protocol called MADD (Mobile Agent-based Directed Diffusion), which is a combination of two other protocols called directed diffusion, and mobile agent (MA)-based distributed sensor network (MADSN). According to MADD, a mobile agent is used for efficient delivery of data to target sensors. Collaborative signal and information technique of MADSN would reduce the sensory data traffic and query in DD, performing which is the objective of this paper. Simulation shows its validity by outperforming DD (in the client/server paradigm) in terms of packet delivery ratio, energy consumption, and end-to-end delivery latency.

\subsection{Multi-agent System for Directed Diffusion in Wireless Sensor Networks [20]}

The protocol proposed here is called AbDD (Agent based Directed Diffusion) that focuses on the increment of the life time of node in wireless sensor networks. Considering the bandwidth wastage done by sensors, AbDD introduces mobile agents to reduce the communication cost and unnecessary use of bandwidth by moving the processing function to the data rather than bringing the data to a central processor (sink). This system has both stationary agent that monitors the events associated with a sensor node during communication and builds its knowledge, as well as mobile agent that travel and interact with stationary agents on each node in WSN. It benefits from the knowledge acquired by stationary agents to choose next best hop towards it destination. This protocol considers the minimum route cost and maximum battery level of node's neighbor to determine its next hop. This enables balanced energy consumption. 
International Journal of Wireless \& Mobile Networks (IJWMN) Vol. 6, No. 3, June 2014

\subsection{Nodes' Credit based Directed Diffusion for wireless sensor networks [21]}

It is an enhanced version of DD which focuses on the improvement of the energy efficiency and reliability of basic DD. It puts emphasis on the nodes' failure as well as effective data dissemination criteria. It has boosted DD by implementing a feature called node's credit. This feature would be considering five factors to make the data aggregation system more trustworthy.A distinctive step taken in this paper is to identify the limitations of basic DD. Considering those limitations, this paper has implemented such algorithm that would be mitigating the problems associated with DD. The algorithm selects an appropriate route for data transmission by considering desired parameters which are number of successful or unsuccessful transmissions, number of existence processing loads in candidate node, amount of energy in selective node's battery, location, and the distance between selective candidate node and destination node (sink) and number of sources that candidate node covers. Evaluation study has proved its better efficiency.

\subsection{On The Performance Of Directed Diffusion In Dense Sensor Networks [22]}

This is a review paper which criticizes on directed diffusion. It analyzed the basic directed diffusion to point out what are the disadvantages in that. Such as flooding which is an expensive protocol followed in DD for interest propagation that requires a lot of energy consumption. It might decrease the performance is case of densely deployed wireless sensor network. Similar to this, this paper precisely identifies DD's weaknesses in such application scenarios, in order to allow suitable optimizations for better performance and future scope of research showing what are the sectors need improvements.

\subsection{RDRT: A Novel Roaming Agent-based Directed Routing Tree Algorithm [23]}

This paper proposed an algorithm called RDRT (Roaming agent-based Directed Routing Tree), which optimizes the basic Directed Diffusion. It follows the basic rules as flooding policy but in addition assigns a routing-repair agent to roam among sensors randomly. This agent identifies the invalid nodes and repairs the routing process by following a repairing process. Simulation results proves that RDRT algorithm can repair the invalid routing path quickly and the energy of sensor node to increase the life of WSN.

\subsection{Reduction of Exploratory Data Messages on Directed Diffusion in Mobile Wireless Sensor Networks [24]}

This paper deals with energy. In the interest propagation phase of basic directed diffusion, the reception of exploratory data is a stage where energy is consumed to due to sensing of the sensor nodes. So this protocol is called EDR (Exploratory Data Reduction). This protocol takes in consideration that reduction of exploratory data facilitates less energy consumption. EDR reduces the number of times to send the exploratory data messages in proportion to the desirable number of routing paths. However, original directed diffusion ensures robustness by delivering exploratory data to all the neighbors of the source node, which is, according to EDR, not necessarily appropriate for all situations. So this protocol selects only some neighbors of the source node for sending the exploratory data. Simulation showed that EDR is less energy consuming than the basic DD.

\subsection{REEP: data-centric, energy-efficient and reliable routing protocol for wireless sensor networks [25]}

The REEP (Reliable and Energy Efficient Protocol) provides an improvement in selecting paths for reliable and energy efficient data delivery process. It uses an 'energy threshold value' for each 
International Journal of Wireless \& Mobile Networks (IJWMN) Vol. 6, No. 3, June 2014

node to check which node agrees or denies for participating in any further activities. It gives more reliable transmission of any event information or real data. Each node maintains an RPQ (Request Priority Queue), which is a kind of first-in-first-out (FIFO) queue, to track the series of received 'information event' from different neighbors and to select a neighbor with highest priority in order to demand for path setup.

\subsection{RMST: Reliable Data Transport in Sensor Networks [26]}

This paper improves the reliability of data delivery. It emphasizes on both MAC layer and transport layer. It considers implementing reliability in the MAC layer, transport layer, application, and combinations of these. MAC-level reliability is essential to provide hop-by-hop error recovery for the transport layer, route discovery and maintenance. Focusing on these issues, RMST (Reliable Multi-Segment Transport) algorithm is developed to provide a new format for transport layer. This is to benefit the in-network data processing for reliable data transfer. RMST benefits from diffusion routing by including minimal additional control traffic.This protocol guarantees delivery even when multiple hops show very high error rates.

\subsection{RTDD: A Real-Time Communication Protocol for Directed Diffusion [27]}

This protocol deals with the real-time issue, which is another important side to get an efficient wireless sensor networks. It proposes RTDD (Real-Time Directed Diffusion) in order to facilitate on-time packet delivery. Implementing some extensions to the basic directed diffusion, it has applied a prioritized scheduling policy over diffusion. It also provides new policies which do not require location knowledge for on-time efficient data delivery.

\subsection{Secure Diffusion for Wireless Sensor Networks [28]}

It's an improvement of the basic directed diffusion paradigm in terms of security. Here considering some compromised/malicious node, a protocol is developed so that the routing algorithm maintains a secured network. The protocol is called secure diffusion which is new security technique called location-binding keys. It ponders the available end-to-end feedback loop in directed diffusion platform. By doing this, the sink can easily differentiate between fabricated data and the original data and select an authentic path for reinforcement. The simulation showed that secure diffusion is capable to deliver authentic data.

\subsection{Secure Directed Diffusion Routing Protocol for Sensor Networks using the LEAP Protocol [29]}

This protocols talks about the security issue in wireless sensor networks. It points on what are the reasons that conventional security protocols are not sensible to apply. It also states that regular key managing protocols are energy consuming for the resource limited sensors. Keeping focus on this, the authors have modified an existing security protocol called LEAP on the platform of directed diffusion. By simulation study they have shown that this protocol can protect the network from malicious outside attacks.

\subsection{Source Routing Directed Diffusion in Wireless Sensor Network [30]}

In this paper, a protocol has been proposed to minimize the energy consumption in the basic directed diffusion. The protocol is called Source Routing Directed Diffusion (SR-DD) in wireless sensor networks. In this protocol, the sink chooses one path from various possible options depending on the residual energy of the intermediate nodes. The sink will be choosing one path from several paths that has intermediate nodes requiring minimum total transmission energy. If all paths have nodes with low energy then a max-min residual energy path is chosen which manages to keep a balance between minimum transmission energy and the energy consumption. 
Simulation study has proved that this protocol outperforms the basic directed diffusion in energy utilization.

\subsection{LKHW: A Directed Diffusion-Based Secure Multicast Scheme for Wireless Sensor Networks [31]}

This paper is an extension of Logical Key Hierarchy (LKH), which is embedded in a directed diffusion based network. The objective is to implement security. Creating a mechanism for secure group communication develops this algorithm. This algorithm provides features like robustness in routing, and security according to established concepts of secure multicast. However this algorithm causes an energy cost that scales roughly logarithmically with the group size; it ensures security by balancing energy consumption.

\section{FEATURES OF THE DIRECTED DIFFUSION ALGORITHMS}

In the previous section, we have discussed about 31 papers related to directed diffusion algorithm. These papers cover various features of wireless sensor networks. The following figure, we categorized them in seven areas: energy, node mobility, security, data transmission, reliability, application, and lifetime.

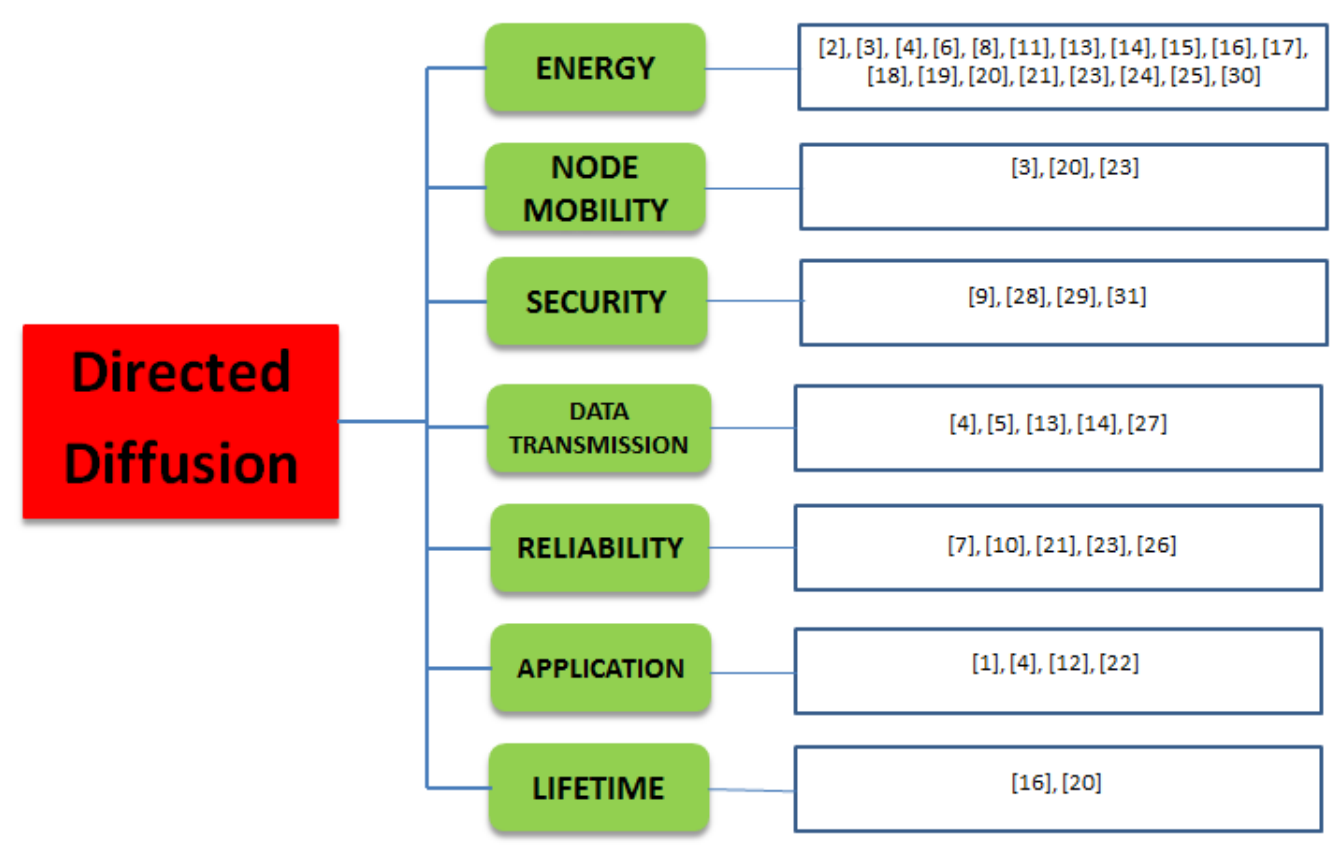

\subsection{Energy}

The following table presents the protocols that enhanced the energy efficiency of directed diffusion protocol. Some important features presented in the table are defined below-

1. Path selection (PS): This feature allows the protocol to select an optimum path considering some parameters unlike directed diffusion where every path is considered.

2. Modification of broadcast phase (MBP): Some other procedure is used instead of general flooding procedure as in basic directed diffusion in the message/interest broadcasting phase. 
International Journal of Wireless \& Mobile Networks (IJWMN) Vol. 6, No. 3, June 2014

3. Residual energy in node (REN): Residual energy of nodes is considered to select intermediate nodes to choose a path.

4. Combination of algorithm (CoA): Shows what algorithms are utilized.

5. Scalability: It means the protocol is capable to adopt with the scaling of the network.

6. Gradient establishment (GE): This feature shows how an algorithm improves the way of gradient establishment in basic directed diffusion during data transmission.

7. Reliability: The protocol ensures proper and reliable data transmission and reception.

8. Node Mobility: In case of any mobile node (could be sink or source or any intermediate node or agent node) the protocol supports the network to keep working.

\begin{tabular}{|c|c|c|c|c|c|c|c|c|c|c|}
\hline 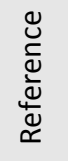 & 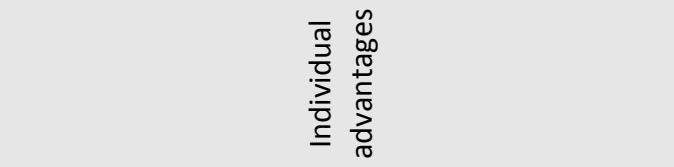 & $\begin{array}{l}\overline{0} \\
\text { o } \\
\text { to } \\
\text { o }\end{array}$ & $\approx$ & $\stackrel{\mathrm{o}}{\stackrel{\underline{m}}{\Sigma}}$ & $\underset{\sim}{\underset{\sim}{u}}$ & త্ & 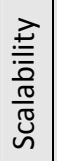 & 岕 & 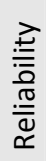 & 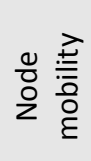 \\
\hline [2] & $\begin{array}{l}\text { 1. Compatible with cluster based network } \\
\text { 2. Energy consumption in MIMO layer (physical } \\
\text { layer) is reduced }\end{array}$ & & & & & $\sqrt{ }$ & & $\sqrt{ }$ & & \\
\hline [3] & $\begin{array}{l}\text { 1. Gradient model which considers- } \\
\text { a. Real-time requirement } \\
\text { b. the numbers of hops from sink to an } \\
\text { intermediate node }\end{array}$ & 足 & $\mathrm{V}$ & & $\mathrm{V}$ & & & $\sqrt{ }$ & $\sqrt{ }$ & V \\
\hline [4] & $\begin{array}{l}\text { 1. Compatible for hierarchical network } \\
\text { 2. Embedded principle of parent selection } \\
\text { 3. Hierarchy can keep the network renewable } \\
\text { and scalable. }\end{array}$ & & & & & $\sqrt{ }$ & $\sqrt{ }$ & & $\sqrt{ }$ & 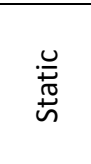 \\
\hline [6] & $\begin{array}{l}\text { 1. Reduce the energy consumption in the } \\
\text { network when there is anoccurrence of failures. } \\
\text { 2. Can repair broken paths by constructing a new } \\
\text { routing tree using a geo-cast approach. }\end{array}$ & & $\mathrm{V}$ & & & & & & 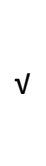 & \\
\hline [8] & $\begin{array}{l}\text { 1. Routing metric is introduced for selection of } \\
\text { intermediate nodes } \\
\text { 2. Metric considers remaining energy of node, } \\
\text { buffer capacity, transmission delay and link } \\
\text { quality }\end{array}$ & & $\mathrm{V}$ & & $\mathrm{V}$ & & & & & \\
\hline [11] & $\begin{array}{l}\text { 1. Threshold } \\
\text { control property that considers limited sensor } \\
\text { resource. } \\
\text { 2. Resolves ill-balanced energy consumption } \\
\text { among sensor nodes. }\end{array}$ & 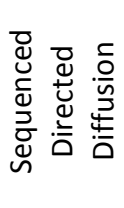 & $\mathrm{V}$ & & V & & & & & \\
\hline [13] & $\begin{array}{l}\text { 1. Reduces the maintenance overheadassociated } \\
\text { with multiple paths } \\
\text { 2. Increases resilience to node failure }\end{array}$ & & $\mathrm{V}$ & $\mathrm{V}$ & & & & & & 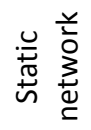 \\
\hline [14] & $\begin{array}{l}\text { 1. Gradient selection considers the number of } \\
\text { hops from the sink } \\
\text { to an intermediate node synchronously }\end{array}$ & & $\mathrm{V}$ & & V & & & $\sqrt{ }$ & & 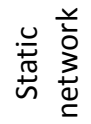 \\
\hline
\end{tabular}


International Journal of Wireless \& Mobile Networks (IJWMN) Vol. 6, No. 3, June 2014

\begin{tabular}{|c|c|c|c|c|c|c|c|c|c|c|}
\hline [15] & $\begin{array}{l}\text { 1. Offers distance-based andcounter-based } \\
\text { broadcast schemes } \\
\text { 2. More energy-balancing than other } \\
\text { schemes } \\
\text { 3. Longer network lifetime than other schemes. }\end{array}$ & 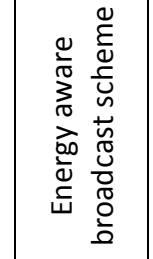 & & & $\sqrt{ }$ & & $\sqrt{ }$ & & & \\
\hline [16] & $\begin{array}{l}\text { 1. The source node } \\
\text { which has the highest residual energy is chosen } \\
\text { as the root }\end{array}$ & $\begin{array}{l}\frac{1}{0} \\
\stackrel{0}{0} \\
\text { मे } \\
\dot{u}\end{array}$ & $\sqrt{ }$ & & $\sqrt{ }$ & & & & & 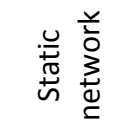 \\
\hline [17] & $\begin{array}{l}\text { 1. Proposes an ant colony algorithm the } \\
\text { foundation of which is gradients based on } \\
\text { pheromone of ants and remnant energy of } \\
\text { nodes. }\end{array}$ & 足 & & & $\sqrt{ }$ & $\sqrt{ }$ & $\sqrt{ }$ & v & & \\
\hline [18] & 1. Provides a path switching mechanism & $0 \overline{0} \stackrel{+}{4}$ & $\sqrt{ }$ & & & & & & & \\
\hline [19] & 1. On time packet delivery & 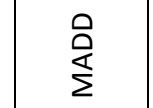 & & & & $\sqrt{ }$ & & & $\sqrt{ }$ & \\
\hline [20] & 1. Agent-based & $\begin{array}{l}\text { 只 } \\
\text { 遂 }\end{array}$ & v & & $\sqrt{ }$ & & & & & 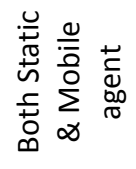 \\
\hline [21] & $\begin{array}{l}\text { 2. Solution to what to do in case of node failure } \\
\text { 2. Finds out the limitations in } D D\end{array}$ & & & & V & & & & $\sqrt{ }$ & 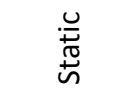 \\
\hline [23] & $\begin{array}{l}\text { 1.Rroaming } \\
\text { agent-based } \\
\text { 2. Repair the } \\
\text { invalid routing path quickly }\end{array}$ & 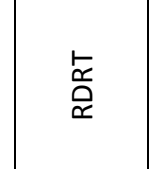 & $\sqrt{ }$ & & & & & & & 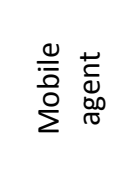 \\
\hline [24] & $\begin{array}{l}\text { 1. Reduced exploratory data } \\
\text { 2. The number of spare routingpaths can be set } \\
\text { in this algorithm. }\end{array}$ & 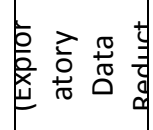 & V & $\sqrt{ }$ & & & & & & \\
\hline [25] & $\begin{array}{l}\text { 1.Makes sensor nodes establish more reliable } \\
\text { and energy-efficient paths. } \\
\text { 2. Considers energy in nodes by checking a } \\
\text { threshold value. }\end{array}$ & 岀 & $\mathrm{V}$ & & V & & & & $\sqrt{ }$ & \\
\hline [30] & $\begin{array}{l}\text { 1. Offers energy equivalence in the network } \\
\text { 2. Avoids local hotspot }\end{array}$ & $\begin{array}{l}0 \\
0 \\
\frac{1}{n}\end{array}$ & $\mathrm{~V}$ & & $\sqrt{ }$ & & & & & \\
\hline
\end{tabular}

\subsection{Security}

The following table presents the protocols that enhanced the security in directed diffusion protocol. Some important features presented in the table are defined below-

1. Data authentication: The receiver acknowledges that the actual message is received. It provides authentication of data delivery.

2. Data integrity: Ensures trustworthiness of the data transmission. 
3. Use of key: The algorithm is using some key for security purpose.

4. Reliability: It refers that actual data is sent to the destination.

5. Dissipated energy: It states how much energy is consumed for the establishment of security.

6. Average delay: It states how data transmission is delayed while ensuring security mechanism.

7. Packet dropping ratio: It shows the state, as sometimes packets are lost while during data transmission.

8. Path selection: Security mechanism is implemented by considering better path selection.

9. Network Mobility: Shows whether the algorithm supports the network in case of mobile nodes.

10. Combination of algorithms: It states what are the other algorithms used to develop the protocol.

\begin{tabular}{|c|c|c|c|c|c|c|c|c|c|c|c|}
\hline 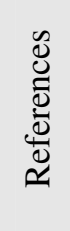 & 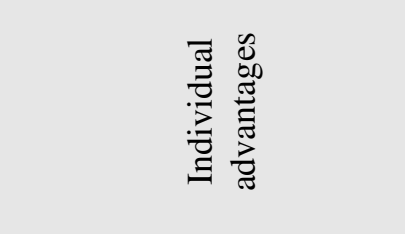 & ص. & 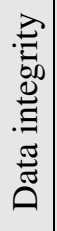 & $\begin{array}{l}\text { ते } \\
\frac{1}{4} \\
0 \\
0 \\
0\end{array}$ & 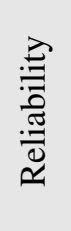 & 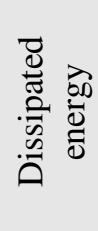 & 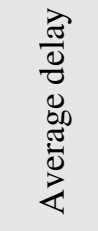 & 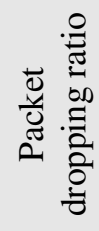 & $\tilde{n}$ & 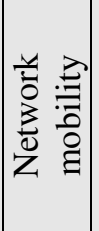 & $\overleftarrow{\delta}$ \\
\hline [9] & $\begin{array}{l}\text { 1. Better event-delivery } \\
2 \text {. Attains better } \\
\text { network performance from } \\
\text { both routing and security } \\
\text { points }\end{array}$ & $\sqrt{ }$ & $\sqrt{ }$ & & $\sqrt{ }$ & 趸 & 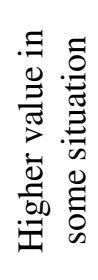 & 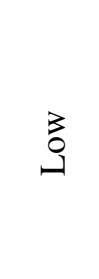 & & & 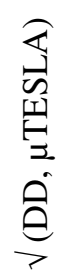 \\
\hline [28] & $\begin{array}{l}\text { 1. Utilizes a novel security } \\
\text { primitive called location- } \\
\text { binding } \\
\text { keys }\end{array}$ & & & $\sqrt{ }$ & & & & & $\sqrt{ }$ & & \\
\hline [29] & $\begin{array}{l}\text { 1. Identifies types of attack } \\
\text { possible for DD } \\
\text { 2.Implementation of } \\
\text { symmetric key algorithm } \\
\text { (LEAP) }\end{array}$ & & $\sqrt{ }$ & $\sqrt{ }$ & & & & & & 芜 & 空 \\
\hline [31] & 1. Robustness & $\sqrt{ }$ & $\sqrt{ }$ & $\sqrt{ }$ & & & & & & & \\
\hline
\end{tabular}

\subsection{Mobility}

The following table mentions about sensor node mobility. These are improved versions of directed diffusion considering if nodes are mobile in network. This table provides the issues when the mobile network is improved.

\begin{tabular}{|c|c|c|c|}
\hline Reference & $\begin{array}{l}\text { Features (what side of } \\
\text { DD is focused to } \\
\text { improve) }\end{array}$ & $\begin{array}{c}\text { Name of the } \\
\text { protocol }\end{array}$ & Consideration \\
\hline [3] & $\begin{array}{l}\text { 1. Gradient model } \\
\text { which considers- } \\
\text { a. Real-time } \\
\text { requirement } \\
\text { b. the numbers of }\end{array}$ & $\begin{array}{c}\text { MEDD } \\
\text { (Mobility and } \\
\text { Energy-Efficient } \\
\text { based on } \\
\text { Directed }\end{array}$ & $\begin{array}{c}\text { Energy efficiency is increased by } \\
\text { considering any node could be } \\
\text { mobile }\end{array}$ \\
\hline
\end{tabular}


International Journal of Wireless \& Mobile Networks (IJWMN) Vol. 6, No. 3, June 2014

\begin{tabular}{|c|l|c|c|}
\hline & $\begin{array}{l}\text { hops from sink to an } \\
\text { intermediate node }\end{array}$ & Diffusion) & \\
\hline$[20]$ & 1. Agent-based & AbDD & $\begin{array}{c}\text { Introducing mobile agent to } \\
\text { improve data transmission facility }\end{array}$ \\
\hline$[23]$ & $\begin{array}{l}1 . \text { Rroaming } \\
\text { agent-based } \\
\text { 2.Repair the } \\
\text { invalid routing path } \\
\text { quickly }\end{array}$ & RDRT & $\begin{array}{c}\text { Lifetime and energy efficiency is } \\
\text { increased by introducing roaming } \\
\text { agent based tree network } \\
\text { construction }\end{array}$ \\
\hline
\end{tabular}

\subsection{Reliability}

The following table mentions the papers discussing about network reliability. Considering what issues the reliability of directed diffusion is improved are described in the following table.

\begin{tabular}{|c|c|c|}
\hline $\begin{array}{l}\text { Reference } \\
\text { Number }\end{array}$ & $\begin{array}{l}\text { Features (what side of DD is } \\
\text { focused to improve) }\end{array}$ & Consideration \\
\hline [7] & $\begin{array}{l}\text { 1.Llink quality was used to } \\
\text { determine an alternative route } \\
\text { that } \\
\text { provides reliable data } \\
\text { transmission } \\
\text { 2. Radio-awareness }\end{array}$ & $\begin{array}{l}\text { Improves directed diffusion by doing optimum } \\
\text { selection of alternative route and radio } \\
\text { awareness }\end{array}$ \\
\hline [10] & $\begin{array}{l}\text { 1. Deals with the communication } \\
\text { reliability } \\
\text { of the network } \\
\text { 2. Does not address the } \\
\text { reliability of the } \\
\text { sensor node or the correctness of } \\
\text { the data collected through } \\
\text { the sensing unit }\end{array}$ & $\begin{array}{l}\text { Self-stabilizing (system will converge to the inten } \\
\text { behavior in finite time, regardless of the system } \\
\text { starting state) }\end{array}$ \\
\hline [21] & $\begin{array}{l}\text { 1. Solution to what to do in case } \\
\text { of node failure } \\
\text { 2. finds out the limitations in DD }\end{array}$ & $\begin{array}{l}\text { Improved directed diffusion considering number } \\
\text { of successful or unsuccessful transmissions, } \\
\text { number of existing processing loads in candidate } \\
\text { node, amount of energy in selective node's } \\
\text { battery, location and the distance between } \\
\text { selective candidate node and destination node } \\
\text { (sink) and numberof sources that candidate node } \\
\text { covers. }\end{array}$ \\
\hline
\end{tabular}


International Journal of Wireless \& Mobile Networks (IJWMN) Vol. 6, No. 3, June 2014

\begin{tabular}{|c|c|c|}
\hline [23] & 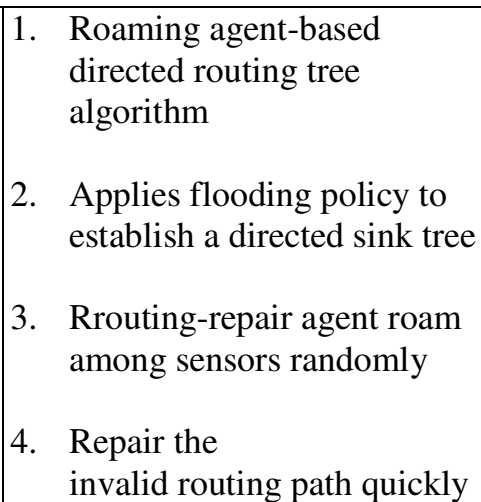 & $\begin{array}{l}\text { Offering facility for path repairing while } \\
\text { implementing roaming agent. }\end{array}$ \\
\hline [26] & $\begin{array}{ll}\text { 1. } & \text { Compatible to multi-layer } \\
\text { network } \\
\text { 2. } & \begin{array}{l}\text { Ensures reliability of data } \\
\text { delivery }\end{array}\end{array}$ & Introducing a new transport layer \\
\hline
\end{tabular}

\subsection{Data Transmission}

The following table mentions the papers concentrates on improving thedata transmission criteria in directed diffusion based wireless sensor network. Considering what issues the data transmission is improved is described in the following table.

\begin{tabular}{|c|c|c|c|}
\hline $\begin{array}{l}\text { Refe } \\
\text { rence }\end{array}$ & Features (what side of DD is focused to improve) & $\begin{array}{c}\text { Name of the } \\
\text { protocol }\end{array}$ & Consideration \\
\hline [4] & $\begin{array}{l}\text { 1. Hierarchical aggregation technique } \\
\text { 2. Save transmission energy to } 50 \% \text { over } \\
\text { Directed Diffusion } \\
\text { 3. Principle of parent selection } \\
\text { 4. Higher reliability }\end{array}$ & $\begin{array}{l}\text { Hierarchical } \\
\text { scheme with } \\
\text { enhancement on } \\
\text { Directed } \\
\text { Diffusion }\end{array}$ & $\begin{array}{c}\text { Network Hierarchy } \\
\text { (Distributes nodes in } \\
\text { hierarchy) }\end{array}$ \\
\hline$[5]$ & $\begin{array}{l}\text { 1. DD + Rumor Routing } \\
\text { 2. Performance of the protocol depends on the } \\
\text { value of the hop count field } \\
\text { 3. Limited flooding } \\
\text { 4. Fewer probability of missing events } \\
\text { 5. Adaptive hop count readjustment } \\
\text { 6. Maximum Utilization of gradients }\end{array}$ & Hybrid Protocol & Hop count value \\
\hline$[13]$ & $\begin{array}{l}\text { 1. Increase in energy efficiency and resilience } \\
\text { 2. Reduces the maintenance overhead } \\
\text { associated with multiple paths } \\
\text { 3. Increases their resilience to node failure }\end{array}$ & & $\begin{array}{l}\text { Improve resilience to path } \\
\text { failure }\end{array}$ \\
\hline$[14]$ & $\begin{array}{l}\text { 1. A new kind of gradient mechanism which } \\
\text { considers residual energy of node and the number } \\
\text { of hops from the sink to an intermediate node } \\
\text { 2. Finds a best-effort optimal path in sensor } \\
\text { networks for data transfer } \\
\text { 3. More globally energy balancing and lower } \\
\text { delay for data transfer than originally DD } \\
\text { 4. Prolongs the lifetime of network efficiently }\end{array}$ & & $\begin{array}{l}\text { Residual energy of node and } \\
\text { the number of hops from the } \\
\text { sink to an intermediate node }\end{array}$ \\
\hline
\end{tabular}


International Journal of Wireless \& Mobile Networks (IJWMN) Vol. 6, No. 3, June 2014

\begin{tabular}{|c|l|l|l|}
\hline$[27]$ & $\begin{array}{l}\text { 1. Improvement in data delivery } \\
\text { 2. Real-time (on time delivery) } \\
\text { 3. Combination of RAP+DD }\end{array}$ & RTDD & Prioritization of packets \\
\hline
\end{tabular}

\subsection{Application}

The following table mentions the papers discussing about application of directed diffusion. Considering what issues the application of directed diffusion is improved are described.

\begin{tabular}{|c|c|c|c|}
\hline $\begin{array}{l}\text { Reference } \\
\text { Number }\end{array}$ & $\begin{array}{l}\text { Features (what side of DD is } \\
\text { focused to improve) }\end{array}$ & $\begin{array}{l}\text { Name of } \\
\text { the } \\
\text { protocol }\end{array}$ & Consideration \\
\hline$[1]$ & $\begin{array}{l}\text { 1. Inspired from the spatial } \\
\text { distribution patterns of insect } \\
\text { populations, which is an } \\
\text { emergent property of the } \\
\text { individual behaviors at the } \\
\text { population level. }\end{array}$ & & $\begin{array}{l}\text { Improved by implementing } \\
\text { mobile agent }\end{array}$ \\
\hline$[4]$ & $\begin{array}{l}\text { 1. Compatible for hierarchical } \\
\text { network } \\
\text { 2. Embedded principle of } \\
\text { parent selection } \\
\text { 3. Hierarchy can keep the } \\
\text { network renewable and } \\
\text { scalable. }\end{array}$ & $\begin{array}{l}\text { Hybrid } \\
\text { Protocol }\end{array}$ & $\begin{array}{l}\text { Improvement of gradient } \\
\text { utilization and flooding }\end{array}$ \\
\hline$\lceil 12]$ & $\begin{array}{l}\text { 1. A general message } \\
\text { machine for routing has been } \\
\text { developed for communication } \\
\text { 2. Compatible for application } \\
\text { environment byswitching } \\
\text { adaptively from normal } \\
\text { model to push model to } \\
\text { match current application } \\
\text { scenarios } \\
\text { 3. With very little changes of } \\
\text { message scripts can make the } \\
\text { routing suitable for a specific } \\
\text { application. }\end{array}$ & ADDRA & $\begin{array}{l}\text { Change of network scenario/ } \\
\text { network condition }\end{array}$ \\
\hline [22] & $\begin{array}{l}\text { 1. Evaluation of the } \\
\text { performance of DD. }\end{array}$ & & Comparative study \\
\hline
\end{tabular}

\subsection{Lifetime}

The following table mentions the papers discussing about lifetime increment of the network. Considering what issues the lifetime of network is increased is described in the following table.

\begin{tabular}{|c|c|c|c|}
\hline $\begin{array}{c}\text { Reference } \\
\text { Number }\end{array}$ & $\begin{array}{c}\text { Features (what } \\
\text { side of DD is } \\
\text { focused to } \\
\text { improve) }\end{array}$ & $\begin{array}{c}\text { Name of the } \\
\text { protocol }\end{array}$ & Consideration/parameter \\
\hline
\end{tabular}


International Journal of Wireless \& Mobile Networks (IJWMN) Vol. 6, No. 3, June 2014

\begin{tabular}{|c|l|l|l|}
\hline$[16]$ & $\begin{array}{l}\text { 1. The source } \\
\text { node } \\
\text { which has the } \\
\text { highest residual } \\
\text { energy is chosen } \\
\text { as the root }\end{array}$ & E-Span & $\begin{array}{l}\text { Residual energy in node } \\
\text { and hop count for } \\
\text { improvement of data } \\
\text { delivery }\end{array}$ \\
\hline$[20]$ & 1. Agent-based & AbDD & $\begin{array}{l}\text { System is improved by } \\
\text { implementing mobile } \\
\text { agents }\end{array}$ \\
\hline
\end{tabular}

\section{CONCLUSIONS}

In this paper, we discussed the various contributions towards the directed diffusion protocol. We have mentioned about 31 research papers to compare these contributions and sorted these features in 7 categories: energy, node mobility, security, data transmission, reliability, application, and lifetime. This study shows that directed diffusion is a very useful and it provides the necessary features. Future study could include in establishing the relationship of the various directed diffusion algorithms.

\section{REFERENCES}

[1] A.W Zhanshan Ma and Krings, "Spatial Distribution Patterns, Power Law, and the agent based directed diffusion for sensor network," in Sixth Annual IEEE International Conference on Pervasive Computing and Communications(PerCom), 2008, pp. 596-601.

[2] Dongfeng Yuan, Yong Wang and Ruihua Zhang Quanquan Liang, "A Distributed Virtual MIMO Scheme Using Cross Layer Design for Wireless Sensor Network," in International Workshop on Cross Layer Design, (IWCLD '07), 2007, pp. 33-36.

[3] Jianrong Wu, Xianghua Xu and Miaoqi Fang Jian Wan, "A Gradient Reconfiguration Mechanism of Directed Diffusion in Wireless Sensor Network," in Fourth International Conference on Semantics, Knowledge and Grid, (SKG '08), 2008, pp. 287-292.

[4] Bu Sung Lee, Cheng Peng Fu and Lek Heng Ngoh Bin Zhou, "A Hierarchical Scheme for Data Aggregation in Sensor Network," in 12th IEEE International Conference on Networks, (ICON 2004), 2004, pp. 525-529.

[5] S.M. Taiabul Haque, "A Hybrid Protocol for Wireless Sensor Network," in First Asian Himalayas International Conference on Internet, (AH-ICI 2009), 2009, pp. 1-3.

[6] H., de Castro, W.L.T. and Filho, R.H. da S Araújo, "A Proposal for Energy Optimization in Wireless sensor networks using an geocast approach," in IEEE 17th International Conference on Telecommunications, (ICT, 2010), 2010, pp. 735-741.

[7] Yong-Jin Park and Euihyun Jung Yong-Pyo Kim, "A Radio-Aware Routing Algorithm for Reliable Directed diffusion in lossy wireless sensor network," Sensors, 2009, vol. 9, no. 10, pp. 8047-8072, October 2009.

[8] A., Jamshidi, K., Mirshams, S. and Rahimizadeh, K. Dehghani, "A routing metric for load balancing considering energy and delay constraints in wireless sensor network," in International Symposium on Telecommunications, (IST 2008), 2008, pp. 745-750.

[9] N., Soliman, O.S., Ghali, N.I., Hassanien, A.E., Palade, V. and Hongbo Liu El-Bendary, "A Secure Directed Diffusion Routing Protocol," in The 2nd International Conference on Next Generation Information Technology, (ICNIT, 2011), 2011, pp. 149-152.

[10] A.K. and Bein, D. Datta, "A Self-Stabilizing Directed Diffusion Protocol for Sensor Networks," in International Conference on Parallel Processing Workshops, (ICPP, 04), 2004, pp. 69-76.

[11] Jaeshin Jang, "A Study on a Sequenced Directed Diffusion algorithms for sensor networks," in The 9th International Conference on Advanced Communication Technology, 2007, pp. 679- 683.

[12] Zeng Zi-ming and Zeng Yuan-yuan Xi Bo, "Adaptive Directed Diffusion Routing in Wireless Sensor Network based on Application," in Canadian Conference on Electrical and Computer Engineering, 2005, pp. 2147- 2150 . 
International Journal of Wireless \& Mobile Networks (IJWMN) Vol. 6, No. 3, June 2014

[13] L.A., Carothers, C.D., and Szymanski, B.K. Bush, "Algorithm for Optimizing Energy Use and Path Resilience in Sensor Networks," in Proceeedings of the Second European Workshop onWireless Sensor Networks, 2005, pp. 391- 396.

[14] Jianrong Wu, Xianghua Xu and Yanna Yan Jian Wan, "An Efficient Gradient Mechanism of Directed Diffusion in wireless sensor network," in International Conference on Computational Intelligence and Security (CIS '08), 2008, pp. 427- 431.

[15] JI Zhen-zhou and HU Ming-zeng Cao Zhi-yan, "An energy-aware broadcast scheme for directed diffusion in wireless sensor network," Journal of Communication and Computer, vol. 4, no. 5, 2007.

[16] M. and Wong, V.W.S. Lee, "An energy-aware spanning tree algorithm for data aggregation in wireless sensor networks," in Pacific Rim Conference on Communications, Computers and signal Processing (PACRIM, 05), 2005.

[17] Li Chunlin and Qian Qiongfen Zhang Xiaoqing, "An Energy Efficient Directed Diffusion Model for wsn," in International Conference on Internet Technology and Applications, 2010, pp. 1-4.

[18] Yuh-Chung Lin, Tsung-Han Chen and Chin-Shiuh Shieh Wei Kuang Lai, "Load-balance Directed Diffusion in Wireless Sensor Networks," in Fifth International Conference on Genetic and Evolutionary Computing (ICGEC,11), 2011, pp. 65-68.

[19] Taekyoung Kwon, Yong Yuan, Yanghee Choi and Victor CM Leung Min Chen, "Mobile AgentBased Directed Diffusion inWireless," EURASIP Journal on Applied Signal Processing, vol. 2007, no. 1, January 2007.

[20] H., Shakshuki, E., Dewolf, T. and Denko, M.K. Malik, "Multi-agent System for Directed Diffusion in Wireless Sensor Networks," in 21st International Conference on Advanced Information Networking and Applications Workshops (AINAW,07), 2007, pp. 635- 640.

[21] Amir Masoud Rahmani and Sam Jabehdari Farnaz Dargahi, "Nodes' Credit based Directed Diffusion for wireless sensor," International Journal of Grid and Distributed Computing (SERSC-08), vol. 1, pp. 39-48, 2008.

[22] L. and Badache, N Khelladi, "On The Performance Of Directed Diffusion In Dense Sensor Network," in 4th International Conference on Innovations in Information Technology (IIT, 07), 2007, pp. 113117.

[23]Chen Xinmeng and Cui Yanrong Li Keqing, "RDRT A Novel Roaming Agent-based Directed Routing Tree algorithm," in International Conference on Wireless Communications, Networking and Mobile Computing (WiCOM, 06), 2006, pp. 1-4.

[24] A. and Teerapabkajomdet, W Booranawong, "Reduction of Exploratory Data Messages on Directed Diffusion in Mobileworks Wireless Sensor Network," in 6th International Conference on Electrical Engineering/Electronics, Computer, Telecommunications and Information Technology (ECTI-CON, 09), 2009, pp. 996- 999.

[25] F., Misra, S., Woungang, I., Rashvand, H.F., Ma, N.-W. and Ahsan Ali, M Zabin, "REEP datacentric, energy-efficient and reliable routing protocol for ws," IET Communications, vol. 2, no. 8, pp. 995-1008, 2008.

[26] F. and Heidemann, J. Stann, "RMST: Reliable Data Transport in Sensor networks," in Proceedings of the First IEEE International Workshop onSensor Network Protocols and Applications , 2003, pp. 102112.

[27] K., Neelisetti, R. and Lim, A. Casey, "RTDD: A Real-Time Communication Protocol for directed diffusion," in IEEE Wireless Communications and Networking Conference (WCNC,08), 2008, pp. 2852-2857.

[28] Wong, S.H.Y., Songwu Lu and Lixia Zhang Hao Yang, "Secure Diffusion for Wireless Sensor Networks," in 3rd International Conference on Broadband Communications, Networks and Systems (BROADNETS, 06), 2006, pp. 1-10.

[29] Johnson Thomas and Ajith Abraham Vijayraman Kumar, "Secure Directed Diffusion Routing ProtocolSecure Directed Diffusion Routing Protocol for Sensor Networks using the LEAP protocol," in Proceedings of the NATO advanced research workshop on information asuurance and computer security, 2006.

[30] Ning hu and Deyun Zhang, "Source routing directed diffusion in wirelss sensor network," Information technology journal, vol. 5, no. 3, pp. 534-539, 2006.

[31] R., Mancini, L.V., Yee Wei Law, Etalle, S. and Havinga, P. Di Pietro, "LKHW: A Directed Diffusion-Based Secure Multicast Scheme forWireless sensor network," in Proceedings. International Conference on Parallel Processing Workshops, 2003, pp. 397-406. 


\section{Authors}

Mohammad Abdus Salam is an Associate Professor in the Department of Computer Science at Southern University, Baton Rouge, Louisiana. He received his BS degree in Electrical and Electronics Engineering from Bangladesh Institute of Technology, Rajshahi in 1991 and M S and Ph.D. degrees from Fukui University, Japan, respectively in 1998 and 2001. Prior to 2005, he worked as an adjunct faculty member of Mathematics and Computer

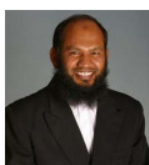
Science at the City University of New York at York College, and as a postdoctoral fellow in the Department of Electrical and Computer Engineering at the University of South Alabama, Mobile, Alabama. $\mathrm{He}$ is a senior member of IEEE. His research interests include wireless communication, error-control coding, and sensor networks.

Tanjima Ferdous is an IT professional. She is currently assigned in a project with Crowley Maritime Corporation, Jacksonville, Florida, in the position of EDI Analyst, responsible for support business requirements of Electronic Data Interchange. She has received her BS degree in Electrical and Electronic Engineering from Ahsanullah University of Science and Technology, Dhaka, Bangladesh in 2010 and MS from Southern University and A\&M College, Baton Rouge, Louisiana in 2013. She did her Masters thesis in Directed Diffusion

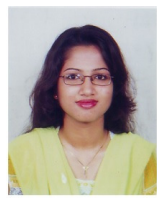
based Secured Aggregation Tree where she developed a concept of a new protocol. She has attended several conferences in Louisiana Academy of Science and achieved best graduate research demonstration award for her work in Southern University Sustainability Week 2013. She keeps interest in programming language, software development and resource management. 